\title{
Supporting Information \\ Optimal self-assembly of linked constructs and catenanes via spatial confinement
}

\author{
Guido Polles ${ }^{1}$, Enzo Orlandini ${ }^{2}$, and Cristian Micheletti ${ }^{1}$ \\ ${ }^{1}$ SISSA, International School for Advanced Studies, \\ via Bonomea 265, I-34136 Trieste, Italy \\ ${ }^{2}$ Dipartimento di Fisica e Astronomia and Sezione INFN, \\ Universita' di Padova, Via Marzolo 8, 35131 Padova, Italy
}




\section{MODEL AND SYSTEM SETUP}

We considered solutions of rigid templates of semi-circular, semi-elliptical and helical shapes composed of touching hard spheres, or beads, of diameter $\sigma$, see Figs. 1a, 3a and 4a.

The model parameters are analogous to those used in the study of self-assembly knots of ref. [1]. Excluded volume effects are accounted for by a Weeks-Chandler-Anderson repulsive potential acting between pairs of hard spheres belonging to different templates:

$$
U^{\mathrm{ex}}(d)=4 \epsilon \kappa_{h s}\left[\left(\frac{\sigma}{d}\right)^{12}-\left(\frac{\sigma}{d}\right)^{6}+\frac{1}{4}\right] \theta\left(2^{1 / 6} \sigma-d\right)
$$

where $d$ is the beads distance, $\epsilon$ is the system energy unit, $\kappa_{h s}=150$ is the interaction strength and $\theta(x)$ is the Heaviside function that takes on the values 1 or 0 for $x$ greater or smaller than 0 , respectively.

The template termini are functionalised with patchy particles whose centers are placed at the inersection of the template centerline with the exposed surface of the terminal beads. Patchy particles interact one to another via the attractive potential:

$$
U^{\mathrm{p}}(d)=-\epsilon \kappa_{p} \exp \left[-\frac{d^{2}}{2 \sigma_{\mathrm{p}}^{2}}\right],
$$

where $\kappa_{p}=25$ and $\sigma_{p}=0.1 \sigma$ are the magnitude and spatial range of the attractive interaction, respectively. The positioning and size of the patchy particles favours the collinear binding of templates[1].

The system was initialised by placing randomly $N=500$ non-overlapping templates inside square slits of height $H$ and side $L$. The slit is periodic in the $x$ and $y$ (unbound) directions and the impenetrability of the slit walls (parallel to the $x y$ Cartesian plane, see Fig. 1b) is enforced by the same potential of eq. 1, where $d$ is the distance along the $z$ axis of the beads and the slit.

For the three cases, we considered monodispersed solutions with the following properties: (i) $N=500$ semi-circular templates of diameter $2 r$ (taken as the unit of length), bead thickness $\sigma=\{1 / 16,1 / 8\}$ placed inside slits of width $H$ in the $[0.5,10]$ range, and at solution densities $\rho \equiv N /\left(H \cdot L^{2}\right)=\{1,2\}$; (ii) $N=500$ semi-elliptical templates with ellipticity (long to short arms) ratio equal to $\chi=\{1,4,9\}$, area equal to $\pi r^{2} / 2$ (again $2 r$ is the unit of length), bead thickness $\sigma=1 / 16$ placed inside slits of height $H$ in the $[0.5,8]$ range, and at solution density $\rho \equiv N /\left(H \cdot L^{2}\right)=1$; (iii) helical templates projecting a circle of diameter $2 r$ 
(taken as the unit length) with vertical span $h$ and angular span $\alpha$, bead thickness $\sigma=1 / 8$, placed inside slits of width $H / 2 r$, in the [1,5] range as well as in bulk. The data in Fig. 5 pertain to a solution of $N=500$ helical templates with $h=0.375$ and $\alpha=1.7 \pi$ at density $\rho \equiv N /\left(H \cdot L^{2}\right)=1$. The representative links and computed probabilities where obtained from systems of $N=250$ unconstrained templates with $h$ in the $[0,0.75]$ range, $\alpha$ in the $[1.5 \pi, 2 \pi]$ range and $\rho$ in the $[2,45]$ range.

\section{DYNAMICAL EVOLUTION}

The system was evolved with a Langevin dynamics and the system energy unit, $\epsilon$ was set equal to the thermal energy $K_{B} T$. The dynamics was integrated with the LAMMPS numerical package[2] with standard parameters for the beads mass, $m$ and friction coefficient, $\gamma$, and integration time step equal to $0.012 \tau_{L J}$, with $\tau_{L J}=\sigma \sqrt{m / \epsilon}[3]$. For each considered combination of system parameters (template geometry, density, slit height) the dynamics was followed over several independent runs, mostly from 20 to 200, and each run typically lasting $2 \cdot 10^{7}$ integration time steps, which suffice to reach a stable concentration of closed constructs for systems of this type[1].

\section{TOPOLOGICAL PROFILING}

Links were identified in two steps. First, each final configuration of the simulations was analysed to identify the rings, i.e. those self-assembled constructs that closed over themselves, and their knotted state based on the Alexander determinants [4]. The rare system configurations featuring constructs propagating "endlessly" through the boundary conditions were discarded. Linked constructs were next identified by computing the two-variable Alexander polynomial $\Delta(s, t)$ evaluated at different pair values $(s, t) \in$ $\{(-1,-1) ;(2,2) ;(2,3) ;(3,2) ;(3,3)\}$ for all pairs of rings. This topological invariant (see, for instance [5] for a justification and [6] for details of the algorithm) can detect homotopycally linked rings, and is more powerful than the linking number which detects the smaller set of homological links [7]. Here, we also used it in conjunction with the knotted state of each linked components, which was established from the single-variable Alexander determinants calculated for each component. The matrix of pairwise homotopycal links is also used 
to identify multi-component links.

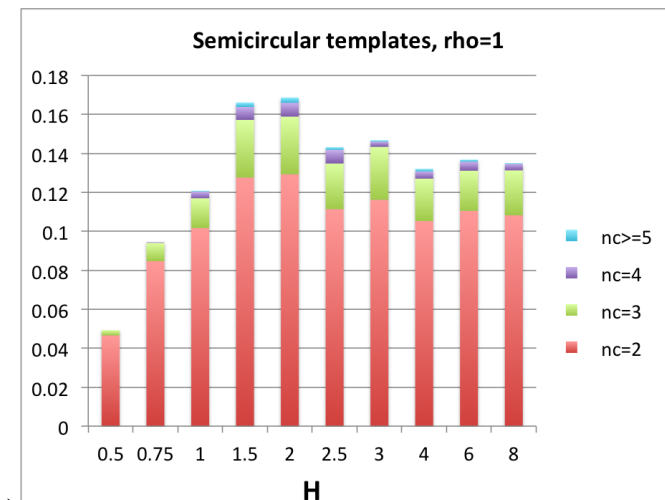

(a)
H

(b)

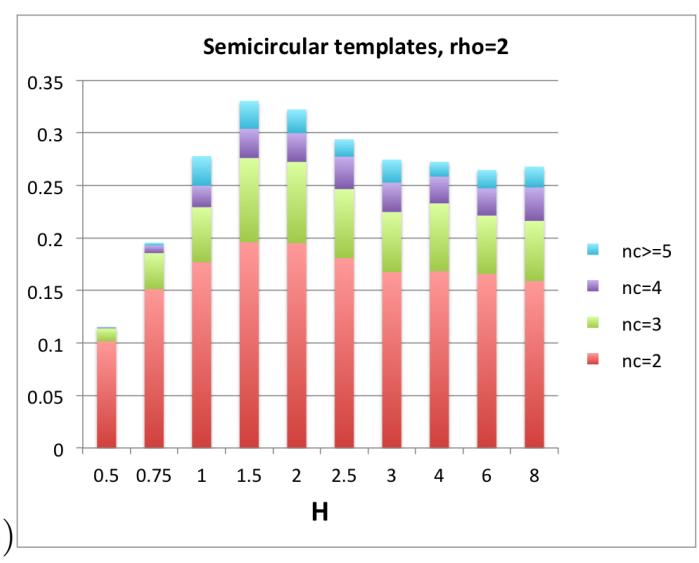

Figure S 1: Relative incidence of non-trivial links with different number of components, $n_{c}$, selfassembled from the semi-circular templates of Fig. 1a and at density $\rho=1$ and $\rho=2$. Note that the data are for the abundance of links and not for the number of involved rings (or templates) which clearly increases with $n_{c}$. Because of the simple geometry of the templates, every pair of homotopically-linked rings in binary or multi-components links has the Hopf topology. 


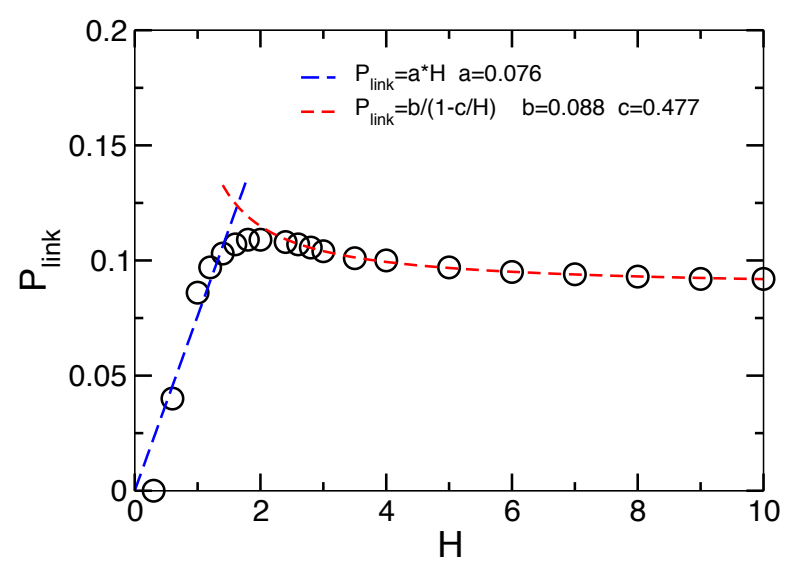

Figure S 2: Linking probability versus slit height for a system of 1000 infinitely thin circular rings at (ring) density $\rho=0.1$ (the rings diameter is taken as the unit of length). The linking probability was computed over $10^{4}$ samples independently generated by randomly placing the rings in the slit and discarding the samples where the rings intersect the boundaries. Notice that the circular rings are pre-formed by construction, and are not self-assembled. The linking probabilities at weak and strong slit confinement are well fitted by the indicated functions based on the arguments presented in the main manuscript. 

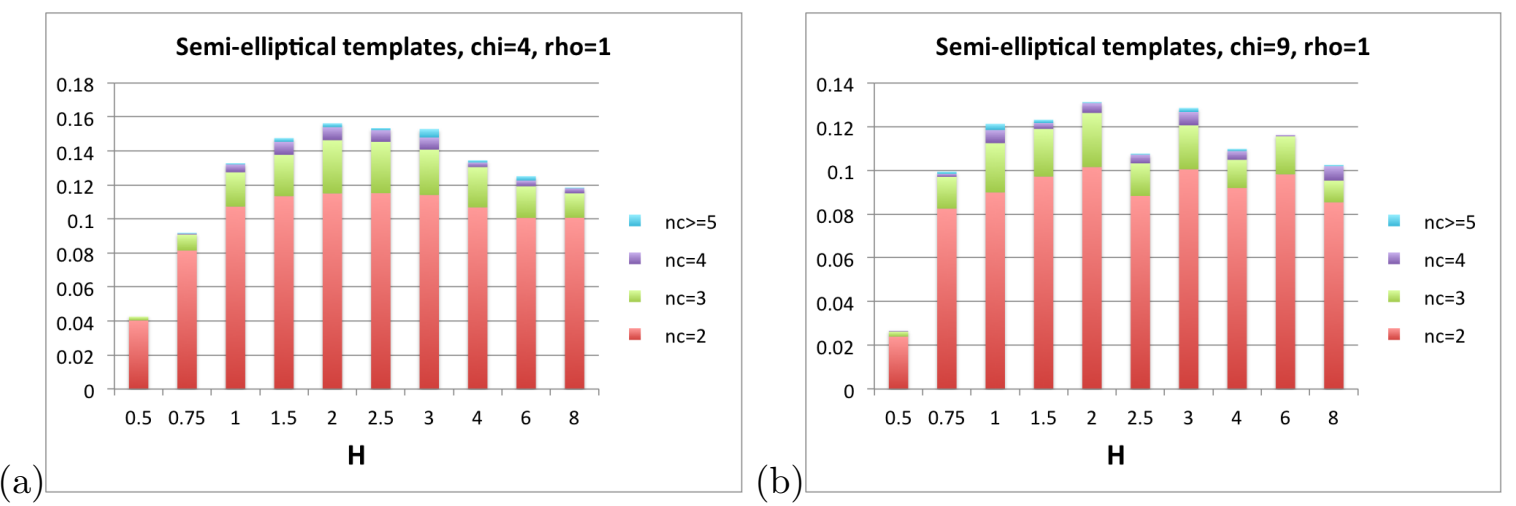

Figure S 3: Relative incidence of non-trivial links with different number of components, $n_{c}$, selfassembled from the semi-elliptical templates of Fig. 3 and at density $\rho=1$ and $\chi=4$ and $\chi=9$. Note that the data are for the abundance of links and not for the number of involved rings (or templates) which clearly increases with $n_{c}$. Because of the simple geometry of the templates, every pair of homotopically-linked rings in binary or multi-components links has the Hopf topology. 


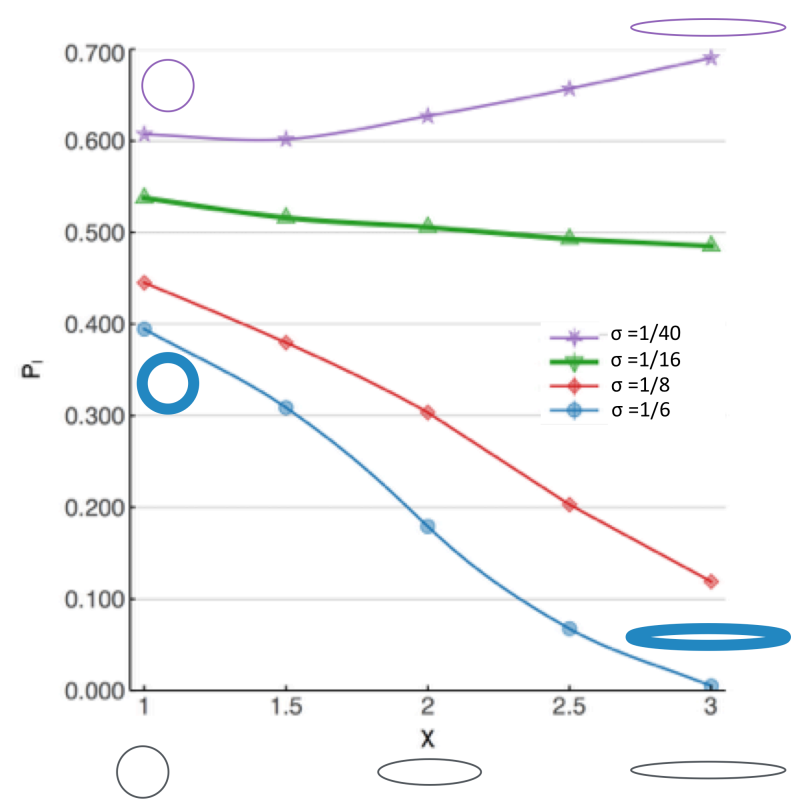

Figure S 4: Effect of template thickness, $\sigma$, on the linking probability of 1000 confined elliptical templates at (ring) density $\rho=1$. The templates geometry is the same as in Fig. 3, but the system samples (at least $10^{4}$ for each data point) were generated starting from preformed (i.e. not self-assembled) elliptical rings with the same stochastic procedures described in the caption of Fig. S2 but complemented with the steric hindrance constraints on the rings. Except for very thin rings, the increase of template thickness brings about a general decrease of the linking probability due to the reduction of the area available for template intersection. 


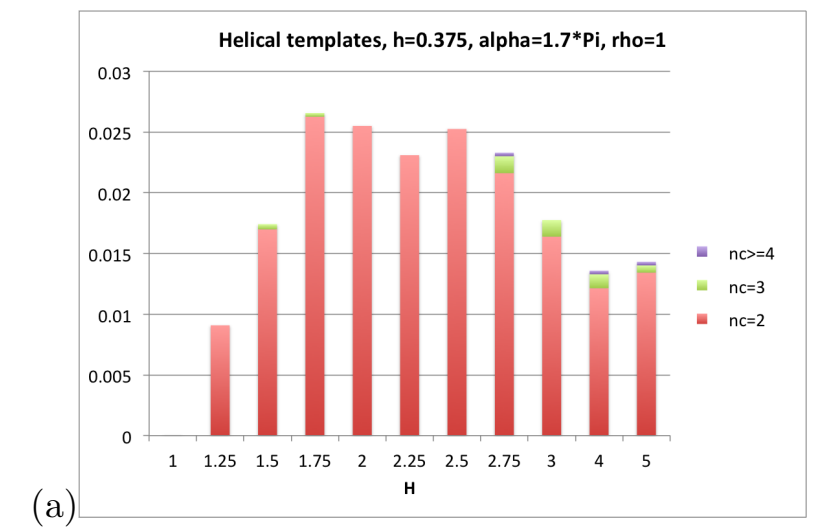

Figure S 5: (a) Relative incidence of non-trivial links with different number of components, $n_{c}$, self-assembled from the helical templates of Fig. 4 and at density $\rho=1$. Note that the data are for the abundance of links and not for the number of involved rings (or templates) which clearly increases with $n_{c}$. 
[1] G. Polles, D. Marenduzzo, E. Orlandini, and C. Micheletti, Nature Communications 6, 6423 (2015).

[2] S. J. Plimpton, J. Comput. Phys. 117, 1 (1995).

[3] K. Kremer and G. Grest, J. Chem. Phys. 92, 5057 (1990).

[4] S. G. W. E. Orlandini, Rev. Mod. Phys. 79, 611 (2007).

[5] G. Torres, Annals Mathematics 57, 57 (1953).

[6] J. Archibald, Ph.D. thesis, University of Toronto (2010).

[7] D. Rolfsen, Knots and Links (Mathematics Lecture Series 7, Publish or Perish, Inc., Houston, Texas, 1976). 